\title{
BOUNDS FOR THE AVAILABILITIES OF MULTISTATE MONOTONE SYSTEMS BASED ON DECOMPOSITION INTO STOCHASTICALLY INDEPENDENT MODULES
}

\author{
J. GÅSEMYR, * University of Oslo
}

\begin{abstract}
Multistate monotone systems are used to describe technological or biological systems when the system itself and its components can perform at different operationally meaningful levels. This generalizes the binary monotone systems used in standard reliability theory. In this paper we consider the availabilities and unavailabilities of the system in an interval, i.e. the probabilities that the system performs above or below the different levels throughout the whole interval. In complex systems it is often impossible to calculate these availabilities and unavailabilities exactly, but it is possible to construct lower and upper bounds based on the minimal path and cut vectors to the different levels. In this paper we consider systems which allow a modular decomposition. We analyse in depth the relationship between the minimal path and cut vectors for the system, the modules, and the organizing structure. We analyse the extent to which the availability bounds are improved by taking advantage of the modular decomposition. This problem was also treated in Butler (1982) and Funnemark and Natvig (1985), but the treatment was based on an inadequate analysis of the relationship between the different minimal path and cut vectors involved, and as a result was somewhat inaccurate. We also extend to interval bounds that have previously only been given for availabilities at a fixed point of time.
\end{abstract}

Keywords: Availability bound; minimal cut vector; minimal path vector; modular decomposition; multistate monotone system

2010 Mathematics Subject Classification: Primary 62N05; 90B25

\section{Introduction}

A multistate monotone system (MMS) $(C, \phi)$ consists of a set $C=\{1,2, \ldots, n\}$ of components and a structure function $\phi$, taking values in the set $S=\{0,1,2, \ldots, M\}$, where $n$ and $M$ are arbitrary natural numbers. The state of component $i$ belongs to a subset $S_{i}$ of $S$, assumed to contain 0 and $M$, and the state at time $t$ is denoted by $X_{i}(t)$. The system state is supposed to be a nondecreasing function of the component states, and is given by $\phi(X(t))$, where, by definition, $X(t)=\left(X_{1}(t), \ldots, X_{n}(t)\right)$. We assume that $\phi(0, \ldots, 0)=0$ and $\phi(M, \ldots, M)=M$. The concept of an MMS generalizes the concept of a binary monotone system (BMS) as treated in Barlow and Proschan (1975). It allows a more refined description of a system than the concept of a BMS, which is often necessary in order to handle complex systems that can perform at different levels. A thorough treatment of multistate monotone systems is given in the book Natvig (2011). The following example is taken from this book, where it is treated in depth. It is presented here in a slightly different form, and will be used to illustrate the different concepts.

Received 9 September 2010; revision received 12 August 2011.

* Postal address: Department of mathematics, University of Oslo, PO Box 1053 Blindern, Oslo, Norway.

Email address: gaasemyr@math.uio.no 
Example 1. (Part (i).) Consider two two-component parallel systems, with binary components $\{1,2\}$ and $\{3,4\}$, respectively, linked together in a series structure. The state space is taken to be $S=\{0,1,2,3\}$. The components are binary; hence, $S_{i}=\{0,3\}, i=1, \ldots, 4$. The structure function is given by

$$
\begin{aligned}
\phi(X)= & I\left(X_{1}+X_{2}=3\right) I\left(X_{3}+X_{4}=3\right)+2 I\left(X_{1}+X_{2}+X_{3}+X_{4}=9\right) \\
& +3 I\left(X_{1}+X_{2}+X_{3}+X_{4}=12\right),
\end{aligned}
$$

where $I(\cdot)$ is the indicator function.

We will illustrate the various concepts that are introduced later by means of this example.

The elements of the state spaces $S$ and $S_{i}$ are thought of as representing an ordering of meaningful performance levels. In specific applications it may be more natural to let $S$ and $S_{i}$ consist of arbitrary real numbers that are directly interpretable as some kind of measurable quantities, but we will not use these kinds of state spaces in this paper.

The component performance processes $\left\{X_{i}(t), t \in I\right\}$ are random, possibly stochastically dependent processes involving repair at fixed or random points of time. A full probabilistic analysis of a multistate monotone system over an interval $I$ requires the specification of a full dynamic model of the joint component process $\{X(t), t \in I\}$. A framework for the specification of such a parametric model is given in Gåsemyr and Natvig (2005). In all but very simple cases analytic calculations are intractable. Gåsemyr and Natvig (2005) outlined a procedure for simulating the process $\{X(t), t \geq 0\}$, and also a data augmentation procedure for using such simulations in Bayesian estimation of the parameters of the model. A program for simulation of a binary system with independent component processes is presented in Huseby et al. (2010), while a similar program for simulation of a multistate system with independent components has been developed; see Huseby and Natvig (2010).

In complex systems, the abovementioned simulation-based probabilistic analysis of the system may be prohibitively costly computationally. In many cases there is also insufficient information to model the dynamic behaviour of the marginal component processes, and even more so the joint process of dependent components. The analysis then has to be based on less accurate information of the system. In this paper we will assume that the component availabilities and unavailabilities to level $j$,

$$
\begin{gathered}
p_{X_{i}}^{j}=\mathrm{P}\left(X_{i}(t) \geq j \text { for all } t \in I\right), \\
q_{X_{i}}^{j}=\mathrm{P}\left(X_{i}(t)<j \text { for all } t \in I\right), \quad i=1, \ldots, n, j=0,1, \ldots, M,
\end{gathered}
$$

are known. The corresponding system availabilities and unavailabilities,

$$
\begin{gathered}
p_{\phi}^{j}=\mathrm{P}(\phi(X(t)) \geq j \text { for all } t \in I), \\
q_{\phi}^{j}=\mathrm{P}(\phi(X(t))<j \text { for all } t \in I), \quad j=1, \ldots, M,
\end{gathered}
$$

can then not be calculated, even in the case of independent components, and we have to resort to upper and lower bounds. In this paper we will focus on lower bounds for the system availabilities at different levels. Lower bounds for unavailabilities are completely analogous. For the binary case, such bounds are studied in Bodin (1970), Esary and Proschan (1970), Barlow and Proschan (1975), and Natvig (1980). The multistate case is considered in Butler (1982), Funnemark and Natvig (1985), and Natvig (1986), (1993). A comprehensive treatment of the area, based also on the results of the present paper, is given in Natvig (2011, Chapter 3). 
The basic bounds given in these publications are based on the sets of minimal path vectors and minimal cut vectors to level $j$, i.e. vectors $\boldsymbol{y}$ and $\boldsymbol{z}$ that are respectively minimal and maximal in the natural ordering on $S_{1} \times \cdots \times S_{n}$ with respect to the properties that $\phi(y) \geq j$ and $\phi(z<j)$, respectively. Such a vector $z$ is called a minimal rather than a maximal cut vector to level $j$ for historical reasons.

Example 2. (Example 1 continued. Part (ii).) To level 3, $(3,3,3,3)$ is the only minimal path vector. There are four minimal cut vectors to level 3 , each having 0 in one place and 3 elsewhere. We use the notation $\left(0_{i}, 3_{C-\{i\}}\right), i=1, \ldots, 4$, for these vectors. These coincide with the minimal path vectors to level 2 . With similar notation, the six minimal cut vectors to level 2 are of the form $\left(0_{i_{1}}, 0_{i_{2}}, 3_{C-\left\{i_{1}, i_{2}\right\}}\right)$. Out of these six vectors, the two vectors $(3,3,0,0)$ and $(0,0,3,3)$ are minimal cut vectors also to level 1 , while the other four are minimal path vectors to level 1 .

Often the system $(C, \phi)$ allows a modular decomposition of the form

$$
\phi(\boldsymbol{x})=\psi(\chi(\boldsymbol{x}))=\psi\left(\chi_{1}\left(\boldsymbol{x}_{A_{1}}\right), \ldots, \chi_{r}\left(\boldsymbol{x}_{A_{r}}\right)\right),
$$

where $A_{1}, \ldots, A_{r}$ is a partition of $C, \boldsymbol{x}_{A_{k}}$ is the vector with components $x_{i}, i \in A_{k}, k=$ $1, \ldots, r, \psi$ is a structure function called the organizing structure function, and $\chi_{1}, \ldots, \chi_{r}$ are structure functions called the modular structure functions.

Example 3. (Example 1 continued. Part (iii).) Define the modular structure functions

$$
\begin{aligned}
& \chi_{1}\left(X_{1}, X_{2}\right)=I\left(X_{1}+X_{2}=3\right)+3 I\left(X_{1}+X_{2}=6\right), \\
& \chi_{2}\left(X_{3}, X_{4}\right)=I\left(X_{3}+X_{4}=3\right)+3 I\left(X_{3}+X_{4}=6\right) .
\end{aligned}
$$

We can then write

$$
\phi(X)=\psi\left(\chi_{1}\left(X_{1}, X_{2}\right), \chi_{2}\left(X_{3}, X_{4}\right)\right)
$$

where

$$
\psi\left(W_{1}, W_{2}\right)=I\left(W_{1} W_{2}=1\right)+2 I\left(W_{1} W_{2}=3\right)+3 I\left(W_{1} W_{2}=9\right) .
$$

With a modular decomposition as given by (3), it is possible to construct bounds for $p_{\phi}^{j}$ by combining bounds for the availabilities of the organizing structure and bounds for the availabilities of the modules, based on the sets of minimal path and cut vectors for $\psi$ and $\chi_{1}, \ldots, \chi_{r}$, respectively. This problem has been considered in Bodin (1970) in the binary case, and in Butler (1982) and Funnemark and Natvig (1985) in the multistate case, with some refinements in Natvig (1986), (1993). All the bounds constructed in these papers build on the common assumption that the processes $\left\{\boldsymbol{X}_{A_{k}}(t), t \in I\right\}$ are stochastically independent in $I$, and we will stick to this assumption throughout this paper. For such bounds to be useful, they must be shown to be advantageous in comparison with the basic bounds based on minimal path and cut vectors for $\phi$, as given in Funnemark and Natvig (1985). A proper understanding of the relationship between the minimal path and cut vectors for the structure functions $\phi, \psi$, and $\chi_{1}, \ldots, \chi_{r}$ is a necessary basis for such a comparison. It turns out that the comparisons made in Butler (1982) are based on an inadequate analysis of this relationship. These shortcomings are inherited in Funnemark and Natvig (1985), who build on the work of Butler (1982). As a result, we have found it necessary to consider some of the results of these papers again.

In this paper we start out in Section 2 by introducing some necessary notation and reviewing the bounds that are relevant to our analysis. In Section 3 we analyse in depth the relationship 
between the minimal path and cut vectors for the different structure functions involved in a modular decomposition. Based on this analysis, we establish assumptions which are shown in Section 5 to ensure that the results of Butler (1982) on lower bounds based on minimal cut vectors are valid. In addition, we extend one of these results, valid when the component processes are independent in $I$, from the availability at a fixed point of time to the availability in an interval. In Section 4 we reprove the results on lower bounds based on minimal path vectors whose proofs in Funnemark and Natvig (1985) rest on the inaccurate results of Butler (1982). An improved bound is obtained in Funnemark and Natvig (1985) by combining the bounds based on minimal path vectors and minimal cut vectors, and in Section 5 we extend to a general interval $I$ a result on this bound stated in the latter paper for the case $I=[t, t]$, again without appropriate sufficient conditions being given.

\section{Notation and basic bounds}

For the sake of readability, we will try to keep the notation as simple as possible, and, hence, deviate to some extent from the notation used in Funnemark and Natvig (1985) and Natvig (2011).

The full notation for the modular decomposition defined by (3) is

$$
\left(\psi,\left(A_{1}, \chi_{1}\right), \ldots,\left(A_{r}, \chi_{r}\right)\right)
$$

but with the partition $A_{1}, \ldots, A_{r}$ implicitly understood; this will often be referred to as $(\psi, \chi)$. The range of the structure functions $\chi_{k}$ may be proper subsets of $S$, denoted by $S_{\chi_{k}}$, always assumed to contain 0 and $M$. For any $j \in S_{\chi_{k}}, j<M$, we define $j^{+}\left(S_{\chi_{k}}\right)=\min \left\{j^{\prime} \mid j^{\prime} \in S_{\chi_{k}}\right.$, $\left.j^{\prime}>j\right\}$. When the state space $S_{\chi_{k}}$ is clear from the context, we often simplify this, and write $j^{+}\left(S_{\chi_{k}}\right)=j^{+}$. We arbitrarily let $M^{+}=M+1$.

We consider availabilities and unavailabilities in a fixed interval $I$, and do not refer to this interval in the notation for the bounds, or in the formulation of the results, unless explicit mention of the interval is needed. This is so in Theorem 3, in which we deal with two different intervals simultaneously. It is also referred to as the interval in the special case $I=[t, t]$ in the formulation of Theorem 4 .

The system availabilities to the different levels are defined in (2) and are collected in the vector

$$
\boldsymbol{p}_{\phi}=\left(p_{\phi}^{1}, \ldots, p_{\phi}^{M}\right)
$$

We use the same notation for the availabilities of the structure functions of the modules and for vectors whose components are these availabilities. These vectors are collected to form the vector

$$
\boldsymbol{p}_{\chi}=\left(\boldsymbol{p}_{\chi_{1}}, \ldots, \boldsymbol{p}_{\chi_{r}}\right) .
$$

This notation is extended down through an increasingly refined hierarchy of modular decompositions to end up at the component level, with the component availabilities $p_{X_{i}}^{j}$ to the different levels $j \in S_{i}$, defined in (1), collected in the vectors $\boldsymbol{p}_{X_{i}}$, again collected in

$$
\boldsymbol{p}_{X}=\left(\boldsymbol{p}_{X_{1}}, \ldots, \boldsymbol{p}_{X_{n}}\right) \text {. }
$$

Unavailabilities, defined by replacing $p$ by $q$ and ' $\geq$ ' by ' $<$ ' in the definitions of the availabilities, are treated similarly.

In order to minimize the notation, throughout this paper, we consider availabilities and unavailabilities for $\phi$ and $\psi$ to a fixed level $j$, representative of any level of interest, and we often drop this $j$ in the notation, especially in the notation related to minimal path and 
cut vectors. For the modules, availabilities and unavailabilities to every level are relevant, but are implicitly included by the notation $\boldsymbol{p}_{\chi_{k}}$ and $\boldsymbol{p}_{\chi}$.

We use the corresponding notation for bounds, e.g.

$$
l_{\chi_{k}}^{* j}, l_{\chi_{k}}^{*}=\left(l_{\chi_{k}}^{* 1}, \ldots, l_{\chi_{k}}^{* M}\right) \text { and } \boldsymbol{l}_{\chi}^{*}=\left(l_{\chi_{1}}^{*}, \ldots, l_{\chi_{r}}^{*}\right) .
$$

Here we refer to the lower bound that, for the system $(C, \phi)$, is defined in terms of the minimal cut vectors $z^{m}, m=1, \ldots, M_{c}$, to level $j$ (see Butler (1982) for the case $I=[t, t]$ and Funnemark and Natvig (1985) for the general case) by

$$
\begin{aligned}
l_{\phi}^{* j} & =\prod_{m=1}^{M_{c}} \mathrm{P}\left(\left(\bigcup_{i=1}^{n}\left(X_{i}(t)>z_{i}^{m}\right)\right) \text { for all } t \in I\right) \\
& =\prod_{m=1}^{M_{c}} \mathrm{P}\left(\left[\max _{1 \leq i \leq n}\left(X_{i}(t)-z_{i}^{m}\right)\right]>0 \text { for all } t \in I\right) .
\end{aligned}
$$

This is a lower bound for $p_{\phi}^{j}$ if the component processes are associated in $I$; see Esary and Proschan (1970) for a definition of the concept of association in $I$.

The basic lower bound using the minimal path vectors $\boldsymbol{y}^{m}, m=1, \ldots, M_{p}$, to level $j$ is given in Funnemark and Natvig (1985) as

$$
l_{\phi}^{\prime \prime j}=\max _{1 \leq m \leq M_{p}} \mathrm{P}\left(\bigcap_{i=1}^{n}\left(X_{i}(t) \geq y_{i}^{m} \text { for all } t \in I\right)\right)=\max _{1 \leq m \leq M_{p}}\left(\mathrm{P}\left(\bigcap_{i=1}^{n}\left(\check{X}_{i} \geq y_{i}^{m}\right)\right)\right),
$$

where, by definition,

$$
\check{X}_{i}=\min _{t \in I}\left(X_{i}(t)\right)
$$

This lower bound is valid regardless of the joint distribution of the component processes.

Bounds (4) and (5) are not determined by the component availabilities (1) alone. Bound (5) is determined by the specification of the joint distribution of $\breve{X}=\left(\breve{X}_{1}, \ldots, \breve{X}_{n}\right)$, and requires the calculation of joint probabilities in this distribution. The calculation of (4) is even more demanding. Such calculations are unrealistic in many cases, and we are then left with simplified versions. Bound (4) takes the form, based on independent component processes (see Butler (1982) and Funnemark and Natvig (1985)),

$$
l_{\phi}^{* * j}\left(\boldsymbol{p}_{\boldsymbol{X}}\right)=\prod_{m=1}^{M_{c}} \coprod_{i=1}^{n} \mathrm{P}\left(\check{X}_{i}>z_{i}^{m}\right)=\prod_{m=1}^{M_{c}} \coprod_{i=1}^{n} p_{i}^{z_{i}^{m}+1},
$$

where we have used the 'ip' operator $\coprod$ defined by

$$
\coprod_{i=1}^{n} p_{i}=1-\prod_{i=1}^{n}\left(1-p_{i}\right)
$$

Note that if $I$ is reduced to a single point $[t, t]$, and if the component states are independent at $t$, then (4) and (7) coincide.

By Funnemark and Natvig (1985), a correspondingly simplified version of (5) gives a valid lower bound under the assumption of associated component processes in $I$ :

$$
l_{\phi}^{\prime j}\left(\boldsymbol{p}_{\boldsymbol{X}}\right)=\max _{1 \leq m \leq M_{p}}\left(\prod_{i=1}^{n} \mathrm{P}\left(\check{X}_{i} \geq y_{i}^{m}\right)\right)=\max _{1 \leq m \leq M_{p}}\left(\prod_{i=1}^{n} p_{i}^{y_{i}^{m}}\right) .
$$

If the component processes are independent in $I$ then (8) is identical to (5). 
Example 4. (Example 1 continued. Part (iv).) Suppose that the components have a common availability $p_{i}^{3}=p$ to level $3, i=1, \ldots, 4$. Since there is only one minimal path vector $(3,3,3,3)$ to level 3 , we have $l_{\phi}^{\prime 3}\left(\boldsymbol{p}_{\boldsymbol{x}}\right)=p^{4}$. This is also the value of $p_{\phi}^{3}$ if the components are independent. Since all minimal path vectors to level 2 have three functioning components, we have $l_{\phi}^{\prime 2}\left(\boldsymbol{p}_{\boldsymbol{x}}\right)=p^{3}$. Similarly, all minimal path vectors to level 1 have two functioning components, and, accordingly, $l_{\phi}^{\prime 1}\left(\boldsymbol{p}_{\boldsymbol{x}}\right)=p^{2}$.

Turning to the $l_{\phi}^{* *}$ bounds, we see that, for each minimal cut vector $z$ to level 3, we have $p_{i}^{z_{i}+1}=p$ for one value of $i$, corresponding to $z_{i}=0$, and $p_{i}^{z_{i}+1}=0$ for the other three values of $i$. Hence, $\bigsqcup_{i=1}^{4} p_{i}^{z_{i}+1}=p$. Since there are four such minimal cut vectors, we have

$$
l_{\phi}^{* * 3}\left(\boldsymbol{p}_{\boldsymbol{x}}\right)=p^{4}=l_{\phi}^{\prime 3}\left(\boldsymbol{p}_{\boldsymbol{x}}\right)
$$

Similarly, for a minimal cut vector $z$ to level 2, we have

$$
\coprod_{i=1}^{4} p_{i}^{z_{i}+1}=1-(1-p)^{2}=2 p-p^{2}
$$

Since there are six such vectors, we have

$$
l_{\phi}^{* * 2}\left(\boldsymbol{p}_{\boldsymbol{x}}\right)=\left(2 p-p^{2}\right)^{6} .
$$

The ordering of this bound and $l_{\phi}^{\prime 2}\left(\boldsymbol{p}_{\boldsymbol{x}}\right)$ depends on the value of $p$. By solving the equation $p^{3}=\left(2 p-p^{2}\right)^{6}$ we see that $p^{3} \geq\left(2 p-p^{2}\right)^{6}$ if and only if $p \in[0,(3-\sqrt{5}) / 2]$. Since the value of $p$ decreases with the length of the interval $I$, the ordering may therefore also depend on the choice of $I$.

There are two cut vectors $(0,0,3,3)$ and $(3,3,0,0)$ to level 1 . This gives $l_{\phi}^{* * 1}\left(\boldsymbol{p}_{\boldsymbol{x}}\right)=$ $\left(2 p-p^{2}\right)^{2}$. This always exceeds $l_{\phi}^{\prime 1}\left(\boldsymbol{p}_{\boldsymbol{x}}\right)=p^{2}$, and, hence, provides a better bound.

Corresponding to these bounds for availabilities, there are bounds for unavailabilities. To present two examples, which also indicate what kind of notation is used in general, we have, parallel to (5) and (8),

$$
\bar{l}_{\phi}^{\prime \prime j}=\max _{1 \leq m \leq M_{c}} \mathrm{P}\left(\bigcap_{i=1}^{n}\left(X_{i}(t) \leq z_{i}^{m}\right) \text { for all } t \in I\right)=\max _{1 \leq m \leq M_{c}}\left(\mathrm{P}\left(\bigcap_{i=1}^{n}\left(\hat{X}_{i} \leq z_{i}^{m}\right)\right)\right)
$$

and

$$
\bar{l}_{\phi}^{\prime j}\left(\boldsymbol{q}_{\boldsymbol{X}}\right)=\max _{1 \leq m \leq M_{c}}\left(\prod_{i=1}^{n} \mathrm{P}\left(\hat{X}_{i} \leq z_{i}^{m}\right)\right)=\max _{1 \leq m \leq M_{c}}\left(\prod_{i=1}^{n} q_{i}^{z_{i}^{m}+1}\right),
$$

where, similar to (6),

$$
\hat{X}_{i}=\max _{t \in I}\left(X_{i}(t)\right) .
$$

Our main focus in this paper is on the availability bounds (7) and (8), which are explicit functions of the vector $\boldsymbol{p}_{\boldsymbol{X}}$. However, we will also treat bounds (4) and (5), both for the sake of completeness and because this is a natural part of the mathematical development.

The principle behind using modular decompositions in connection with availability bounds is that we replace the usually unknown vector of availabilities for modules by a corresponding vector of bounds. For instance, we replace $\boldsymbol{p}_{\chi}$ in the lower bound $l_{\psi}^{* * j}\left(\boldsymbol{p}_{\chi}\right)$ for $p_{\psi}^{j}=p_{\phi}^{j}$ by $\boldsymbol{l}_{\chi}^{* *}$.

Example 5. (Example 1 continued. Part (v).) For the modular structure functions $\chi_{k}, k=1,2$, we have one minimal cut vector $(0,0)$ to level 1 , and, hence,

$$
l_{\chi_{k}}^{* * 1}\left(\boldsymbol{p}_{\boldsymbol{x}}\right)=1-(1-p)^{2}=2 p-p^{2}=: l^{1} .
$$


To level 3, there are two minimal cut vectors $(3,0)$ and $(0,3)$, and, hence,

$$
l_{\chi_{k}}^{* * 3}\left(\boldsymbol{p}_{\boldsymbol{x}}\right)=p^{2}=: l^{3} \text {. }
$$

Focusing now on the functioning level $j=2$ for the system, the minimal cut vectors for the organizing structure function $\psi$ to level 2 are $(3,0),(0,3)$, and $(1,1)$. Consequently,

$$
\begin{aligned}
l_{\psi}^{* * 2}\left(\boldsymbol{l}_{\chi}^{* *}\left(\boldsymbol{p}_{\boldsymbol{x}}\right)\right) & =l^{1} \times l^{1} \times\left(1-\left(1-l^{3}\right)^{2}\right) \\
& =\left(2 p-p^{2}\right)^{2}\left(1-\left(1-p^{2}\right)^{2}\right) \\
& =\left(2 p-p^{2}\right)^{2}\left(2 p^{2}-p^{4}\right) .
\end{aligned}
$$

The corresponding bound based on minimal path vectors are easier to derive. There are two minimal path vectors $(3,1)$ and $(1,3)$ for $\psi$ to level 2 , both giving rise to the same bound

$$
l_{\psi}^{\prime 2}\left(\boldsymbol{l}_{\chi}^{\prime}\left(\boldsymbol{p}_{\boldsymbol{x}}\right)\right)=l_{\chi_{1}}^{\prime 3}\left(\boldsymbol{p}_{\boldsymbol{x}}\right) \times l_{\chi_{2}}^{\prime 1}\left(\boldsymbol{p}_{\boldsymbol{x}}\right)=p^{2} \times p=p^{3} .
$$

Note that this coincides with $l_{\phi}^{\prime 2}\left(\boldsymbol{p}_{\boldsymbol{x}}\right)$.

In Section 4 we will prove (see Theorem 2), as stated in Funnemark and Natvig (1985), that in fact

$$
l_{\psi}^{\prime j}\left(\boldsymbol{l}_{\boldsymbol{\chi}}^{\prime}\left(\boldsymbol{p}_{\boldsymbol{X}}\right)\right)=l_{\phi}^{\prime j}\left(\boldsymbol{p}_{\boldsymbol{X}}\right)
$$

in general, and use this to prove an equality concerning upper bounds (see Theorem 3 ), stated in Funnemark and Natvig (1985) and Natvig (1986).

For the bounds based on minimal cut vectors, it is easy to see that in Example 1 a corresponding equality holds for functioning levels $j=1$ and $j=3$. However, for $j=2$, the bound $\left(2 p-p^{2}\right)^{2}\left(2 p^{2}-p^{4}\right)$ that is based on modular decomposition differs from the bound $\left(2 p-p^{2}\right)^{6}$ that is not. It is an elementary but quite tricky piece of calculus to show that the former is better than the latter. In Section 5 we will prove (see Theorem 5) that, under certain additional assumptions, which are formulated in Theorem 1 and are satisfied in Example 1,

$$
l_{\psi}^{* * j}\left(\boldsymbol{l}_{\boldsymbol{\chi}}^{* *}\left(\boldsymbol{p}_{\boldsymbol{X}}\right)\right) \geq l_{\phi}^{* * j}\left(\boldsymbol{p}_{\boldsymbol{X}}\right) .
$$

This inequality was claimed to hold in Butler (1982) and Funnemark and Natvig (1985), but only for the case of $I=[t, t]$, and without realising the need for additional assumptions. In Natvig and Mørch (2003) the Norwegian offshore gas pipeline system, as of the 1980s, was analysed as an MMS. It was found that (11) is satisfied for $I=[0.5,0.5]$, where time is measured in years. But, for certain functioning levels, the opposite inequality holds with $I=[0.5,0.6]$. Hence, the additional assumptions are in fact needed, and are apparently not satisfied in this case.

\section{Minimal path vectors and cut vectors for the system, the organizing structure, and the modules}

From now on we use the abbreviations MPV for 'minimal path vector' and MCV for 'minimal cut vector'. For MPVs $\boldsymbol{u}=\left(u_{1}, \ldots, u_{r}\right)$ and MCVs $\boldsymbol{v}=\left(v_{1}, \ldots, v_{r}\right)$ for $\psi$ to level $j$, we will have to consider MPVs for $\chi_{k}$ to level $u_{k}$ and MCVs for $\chi_{k}$ to level $v_{k}^{+}\left(S_{\chi_{k}}\right), k=1, \ldots, r$. Since we may have $u_{k}=0$ and $v_{k}=M$, we must deal with path vectors for $\chi_{k}$ to level $0,(0, \ldots, 0)$ obviously being the only minimal one, and cut vectors for $\chi_{k}$ to level $M+1,(M, \ldots, M)$ obviously being the only minimal one.

The following lemma is a key result for the analysis of the availability bounds based on modular decomposition. 
Lemma 1. Let $(\psi, \chi)$ be a modular decomposition of $(C, \phi)$, defined as in (3).

(a) The MPVs for $\phi, \psi$, and $\chi$ are related as follows.

(i) Let $\boldsymbol{y}$ be an MPV for $\phi$ to level $j$. For each $k=1, \ldots, r$, let $j_{k}=\chi_{k}\left(\boldsymbol{y}_{A_{k}}\right)$. Then there exists an MPV $\boldsymbol{u}$ for $\psi$ to level $j$ with $\boldsymbol{u} \leq\left(j_{1}, \ldots, j_{r}\right)$, and, for each such $\boldsymbol{u}$ and each $k=1, \ldots, r, \boldsymbol{y}_{A_{k}}$ is an MPV for $\chi_{k}$ to level $u_{k}$.

(ii) Suppose that, for each $k=1, \ldots, r$ and each $u_{k} \in S_{\chi_{k}}$, every MPV $\boldsymbol{y}_{A_{k}}$ to level $u_{k}$ satisfies $\chi_{k}\left(y_{A_{k}}\right)=u_{k}$. Then the vector $\boldsymbol{u}$ of $(i)$ is unique.

(iii) Make the same assumption as in (ii). Let $\boldsymbol{u}$ be an MPV for $\psi$ to level $j$. For each $k=1, \ldots, r$, let $\boldsymbol{y}_{A_{k}}$ be an MPV for $\chi_{k}$ to level $u_{k}$. Then $\boldsymbol{y}=\left(\boldsymbol{y}_{A_{1}}, \ldots, \boldsymbol{y}_{A_{r}}\right)$ is an MPV for $\phi$ to level $j$.

(b) The MCVs for $\phi$, $\psi$, and $\chi$ are related as follows.

(i) Let $z$ be an MCV for $\phi$ to level $j$. For each $k=1, \ldots, r$, let $j_{k}=\chi_{k}\left(z_{A_{k}}\right)$. Then there exists an $M C V \boldsymbol{v}$ for $\psi$ to level $j$ with $\boldsymbol{v} \geq\left(j_{1}, \ldots, j_{r}\right)$, and, for each such $\boldsymbol{v}$ and each $k=1, \ldots, r, z_{A_{k}}$ is an MCV for $\chi_{k}$ to level $v_{k}^{+}=v_{k}^{+}\left(S_{\chi_{k}}\right)$.

(ii) Suppose that, for each $k=1, \ldots, r$ and $v_{k} \in S_{\chi_{k}}$, every $M C V z_{A_{k}}$ to level $v_{k}^{+}$ satisfies $\chi_{k}\left(z_{A_{k}}\right)=v_{k}$. Then the vector $v$ of $(i)$ is unique.

(iii) Make the same assumption as in (ii). Let $v$ be an MCV for $\psi$ to level $j$. For each $k=1, \ldots, r$, let $z_{A_{k}}$ be an MCV for $\chi_{k}$ to level $v_{k}^{+}$. Then $z=\left(z_{A_{1}}, \ldots, z_{A_{r}}\right)$ is an MCV for $\phi$ to level $j$.

Proof. To prove (a)(i), note that, clearly, $\left(j_{1}, \ldots, j_{r}\right)$ is a path vector for $\psi$ to level $j$. If it is not minimal, we may successively replace the components $j_{k}$ by lower values $u_{k}$ until no further reduction is possible without causing $\psi(\boldsymbol{u})<j$. Then $\boldsymbol{u}$ is an MPV. Let $\boldsymbol{u}$ be any MPV to level $j$ for $\psi$, satisfying $\boldsymbol{u} \leq\left(j_{1}, \ldots, j_{r}\right)$. Then, for each $k=1, \ldots, r, \boldsymbol{y}_{A_{k}}$ is a path vector for $\chi_{k}$ to level $u_{k}$, being in fact a path vector to level $j_{k} \geq u_{k}$. We claim that it is in fact an MPV. To see this, assume for simplicity that $k=1$. If $\boldsymbol{y}_{A_{1}}=(0, \ldots, 0)$ then $u_{1}=0$, and the claim is satisfied. Otherwise, choose an arbitrary $i \in A_{1}$ for which $y_{i}>0$. Let $y_{i}$ be replaced by some $y_{i}^{\prime}<y_{i}$, to give rise to the modified vectors $\boldsymbol{y}_{A_{1}}^{\prime}$ and $\boldsymbol{y}^{\prime}$, leaving all components except $y_{i}$ unchanged. By the minimality of $\boldsymbol{y}$ we then have

$$
\psi(\boldsymbol{u}) \geq j>\phi\left(\boldsymbol{y}^{\prime}\right)=\psi\left(\chi_{1}\left(\boldsymbol{y}_{A_{1}}^{\prime}\right), \chi_{2}\left(\boldsymbol{y}_{A_{2}}\right), \ldots, \chi_{r}\left(\boldsymbol{y}_{A_{r}}\right)\right) \geq \psi\left(\chi_{1}\left(\boldsymbol{y}_{A_{1}}^{\prime}\right), u_{2}, \ldots, u_{r}\right) .
$$

This means that we must have $u_{1}>\chi_{1}\left(\boldsymbol{y}_{A_{1}}^{\prime}\right)$, proving that $\boldsymbol{y}_{A_{1}}$ is an MPV for $\chi_{1}$ to level $u_{1}$. Under the assumption of (a)(ii), the vector $\boldsymbol{u}$ satisfies $u_{k}=\chi_{k}\left(\boldsymbol{y}_{A_{k}}\right)=j_{k}$. Hence, $\boldsymbol{u}=$ $\left(j_{1}, \ldots, j_{r}\right)$. To prove (a)(iii), let $\boldsymbol{u}$ and $\boldsymbol{y}$ be as stated therein. Then, clearly, $\boldsymbol{y}$ is a path vector for $\phi$ to level $j$. Choose $y_{i}^{\prime}<y_{i}$ for some $i$. For simplicity, we may assume that $i \in A_{1}$. Let $\boldsymbol{y}^{\prime}$ and $\boldsymbol{y}_{A_{1}}^{\prime}$ represent the corresponding adjustments of $\boldsymbol{y}$ and $\boldsymbol{y}_{A_{1}}$, as above. Then $\chi_{1}\left(\boldsymbol{y}_{A_{1}}^{\prime}\right)=u_{1}^{\prime}<u_{1}$. For $k \neq 1$, by assumption, $\chi_{k}\left(\boldsymbol{y}_{A_{k}}\right)=u_{k}$. Hence,

$$
\phi\left(\boldsymbol{y}^{\prime}\right)=\psi\left(\chi_{1}\left(\boldsymbol{y}_{A_{1}}^{\prime}\right), \chi_{2}\left(\boldsymbol{y}_{A_{2}}\right), \ldots, \chi_{r}\left(\boldsymbol{y}_{A_{r}}\right)\right)=\psi\left(u_{1}^{\prime}, u_{2}, \ldots, u_{r}\right)<j,
$$

proving that $\boldsymbol{y}$ is an MPV to level $j$. The proof of part (b) is similar and thus omitted.

Remark 1. Lemma 1(a)(ii) is sufficient to obtain uniqueness in the determination of an appropriate $\boldsymbol{u}$, but the vector $\boldsymbol{u}$ may be uniquely determined without it. An example is provided by 
assuming that $\chi_{k}$ satisfies the condition for $k=2, \ldots, r$, while $\chi_{1}$ does not. Then we have $u_{k}=j_{k}$ for $k=2, \ldots, r$, and it follows that $u_{1}$ is uniquely determined, i.e. the conclusion of Lemma 1(a)(ii) holds. However, we are not able to prove that the conclusion of Lemma 1(a)(iii) holds in these circumstances.

Example 6. (Example 1 continued. Part (vi).) Recall that, for the modular structure functions $\chi_{k}, k=1,2$, the state space is $S_{\chi_{k}}=\{0,1,3\}$. For the MPVs $(3,0)$ and $(0,3)$ to level 1 , we have $\chi_{k}(3,0)=\chi_{k}(0,3)=1$. For the single MPV $(3,3)$ to level 3 , we obviously have $\chi_{k}(3,3)=3$. Hence, the condition in Lemma 1(a)(ii) is satisfied. For the single MCV $(0,0)$ for $\chi_{k}$ to level $1=0^{+}$, we have $\chi_{k}(0,0)=0$. Also, for the MCVs $(3,0)$ and $(0,3)$ to level $3=1^{+}$, we have $\chi_{k}(3,0)=\chi_{k}(0,3)=1$. Hence, the condition in Lemma $1(\mathrm{~b})$ (ii) is also satisfied. It is a tedious exercise to verify directly that the conclusions of parts (ii) and (iii) of Lemma 1(a) and (b) are also satisfied.

Butler (1982) tried to characterize the MCVs for $\phi$ by means of binary structure functions related to the MCVs for $\psi$. In our notation, the set of vectors that he claimed to be the distinct MCVs for $\phi$, without any additional assumption on the structure functions $\chi_{k}$, is in fact identical to the set that arises from the construction in Lemma 1(b)(iii). The following example shows that this set may in fact contain replicates of certain cut vectors, and also cut vectors that are not minimal. We demonstrate by this example that, without the assumptions of part (ii) of Lemma 1(a) and (b), the construction in (i) is not necessarily unique, and vectors $\boldsymbol{y}$ and $\boldsymbol{z}$ of the form given in part (iii) of Lemma 1(a) and (b) are not necessarily MPVs and MCVs, respectively.

Example 7. Let $C=\{1,2,3,4\}, A_{1}=\{1,2\}, A_{2}=\{3,4\}, S=\{0, \ldots, 3\}$, and $S_{i}=$ $\{0,1,3\}, i=1, \ldots, 4$. Let the MPVs for $\psi$ be $(3,3)$ to level $3,(3,1),(1,3)$, and $(2,2)$ to level 2 , and $(1,1)$ to level 1 . Let the MPVs for $\chi_{1}=\chi_{2}$ be $(3,1)$ and $(1,3)$ to level $3,(3,1)$ and $(0,3)$ to level 2 , and $(1,1)$ and $(3,0)$ to level 1 . Hence, $(3,1)$ is an MPV both to level 3 and level 2 for $\chi_{k}, k=1,2$. Following the procedure of Lemma 1(a)(iii), with $\boldsymbol{u}=(2,2)$, we obtain a vector $(3,1,3,1)$ which is not an MPV to level 2 , since, e.g. $(3,1,1,1)$ is a path vector to level 2. Hence, the procedure of Lemma 1(a)(iii) does not always give an MPV. On the other hand, $(1,1,1,1)$ is an MCV to level 2 for $\phi$. Following the procedure of Lemma 1(b)(i), we see that $\chi_{1}((1,1))=\chi_{2}((1,1))=1$. We seek an MCV $v \geq(1,1)$ for $\psi$ to level 2 , and observe that both $(2,1)$ and $(1,2)$ meet this requirement. Hence, the procedure of Lemma $1(b)(i)$ does not always give a unique MCV $\boldsymbol{v}$.

By duality, this example also shows that in general the procedure of Lemma 1(a)(i) does not necessarily give a unique MPV $\boldsymbol{u}$ for $\psi$, and that a vector $z$ constructed as in Lemma 1(b)(iii) is not necessarily an MCV for $\phi$.

It is convenient to enumerate the different sets of MPVs and MCVs. This is done in the following definition, in which we also introduce some more useful notation.

Definition 1. Let the MPVs to level $j$ for $\psi$ and $\phi$ respectively be

$$
\left\{\boldsymbol{u}^{l}: l=1, \ldots, L_{p}\right\} \quad \text { and } \quad\left\{\boldsymbol{y}^{m}: m=1, \ldots, M_{p}\right\} .
$$

Also, for each $\boldsymbol{u}^{l}=\left(u_{1}^{l}, \ldots, u_{r}^{l}\right)$ and each $k=1, \ldots, r$, let the MPVs for $\chi_{k}$ to level $u_{k}^{l}$ be

$$
\left\{\boldsymbol{y}_{A_{k}}^{l, s}: s \in\left\{1,2, \ldots, e_{l, k}\right\}=E_{l, k}\right\} .
$$

We denote the components of $\boldsymbol{y}_{A_{k}}^{l, s}$ by $y_{i}^{l, k, s}, i \in A_{k}$. 
Similarly, let the MCVs to level $j$ for $\psi$ and $\phi$ respectively be

$$
\left\{\boldsymbol{v}^{l}: l=1, \ldots, L_{c}\right\} \quad \text { and } \quad\left\{z^{m}: m=1, \ldots, M_{c}\right\} .
$$

Also, for each $\boldsymbol{v}^{l}=\left(v_{1}^{l}, \ldots, v_{r}^{l}\right)$ and each $k=1, \ldots, r$, let the MCVs for $\chi_{k}$ to level $v_{k}^{l+}$ be

$$
\left\{z_{A_{k}}^{l, s}: s \in\left\{1,2, \ldots, b_{l, k}\right\}=B_{l, k}\right\} \text {. }
$$

We denote the components of $z_{A_{k}}^{l, s}$ by $z_{i}^{l, k, s}, i \in A_{k}$.

With these definitions we can now rephrase in the following theorem the main content of Lemma 1, providing a sufficient, operational condition which ensures that there is a one-to-one correspondence between, on the one hand, MPVs $\boldsymbol{y}$ for $\phi$ to level $j$, and, on the other hand, MPVs $\boldsymbol{u}$ for $\psi$ to level $j$ and corresponding MPVs $\boldsymbol{y}_{A_{k}}$ for $\chi_{k}$ to level $u_{k}, k=1, \ldots, r$. The theorem also provides a sufficient, operational condition ensuring that there is a one-to-one correspondence between, on the one hand, MCVs $z$ for $\phi$ to level $j$, and, on the other hand, MCVs $\boldsymbol{v}$ for $\psi$ to level $j$ and corresponding MCVs $z_{A_{k}}$ for $\chi_{k}$ to level $v_{k}^{+}, k=1, \ldots, r$. In turn, this one-to-one correspondence is used in Section 5 to prove inequality (11).

Theorem 1. Let $(\psi, \chi)$ be a modular decomposition of $(C, \phi)$, as defined by (3). Suppose that the following condition is satisfied.

(i) For each $k=1, \ldots, r$ and $u_{k} \in S_{\chi_{k}}$, every MPV $\boldsymbol{y}_{A_{k}}$ to level $u_{k}$ satisfies $\chi_{k}\left(y_{A_{k}}\right)=u_{k}$.

Then, with the notation of Definition 1, we have the following property.

(ii) For each $l \in\left\{1,2, \ldots, L_{p}\right\}$ and each $s \in E_{l, 1} \times \cdots \times E_{l, r}$, there exists $m \in\{1,2, \ldots$, $\left.M_{p}\right\}$ such that

$$
\boldsymbol{y}^{m}=\left(\boldsymbol{y}_{A_{1}}^{l, s_{1}}, \ldots, \boldsymbol{y}_{A_{r}}^{l, s_{r}}\right) .
$$

Conversely, each $\boldsymbol{y}^{m}$ can be written uniquely in this way.

Similarly, suppose that the following condition is satisfied.

(iii) For each $k=1, \ldots, r$ and $v_{k} \in S_{\chi_{k}}$, every $M C V z_{A_{k}}$ to level $v_{k}^{+}$satisfies $\chi_{k}\left(z_{A_{k}}\right)=v_{k}$.

Then, with the notation of Definition 1 , we have the following property.

(iv) For each $l \in\left\{1,2, \ldots, L_{c}\right\}$ and each $s \in B_{l, 1} \times \cdots \times B_{l, r}$, there exists $m \in\left\{1,2, \ldots, M_{c}\right\}$ such that

$$
z^{m}=\left(z_{A_{1}}^{l, s_{1}}, \ldots, z_{A_{r}}^{l, s_{r}}\right)
$$

Conversely, each $z^{m}$ can be written uniquely in this way.

Example 8. (Example 1 continued. Part (vii).) Again, we focus on functioning level 2. The MPV $(3,1)$ for $\psi$ to level 2 corresponds to the MPVs $(3,3,3,0)$ and $(3,3,0,3)$ for $\phi$ to level 2. The MPV $(1,3)$ corresponds to $(3,0,3,3)$ and $(0,3,3,3)$. The MCV $(3,0)$ for $\psi$ to level 2 corresponds to the $\operatorname{MCV}(3,3,0,0)$ for $\phi$ to level 2. Similarly, $(0,3)$ corresponds to $(0,0,3,3)$. Finally, the MCV $(1,1)$ for $\psi$ corresponds to the four MCVs $(3,0,3,0),(3,0,0,3),(0,3,3,0)$, and $(0,3,0,3)$.

With the notation of Definition 1 we can now also write the lower bound based on a modular decomposition introduced in (10) as

$$
l_{\psi}^{\prime j}\left(\mathbf{l}_{\chi}^{\prime}\left(\boldsymbol{p}_{\boldsymbol{X}}\right)\right)=\max _{1 \leq l \leq L_{p}} \prod_{k=1}^{r}\left[\max _{s \in E_{l, k}} \prod_{i \in A_{k}} p_{i}^{y_{i}^{l, k, s}}\right] .
$$


The lower bound based on a modular decomposition introduced in (11) can be written as

$$
l_{\psi}^{* * j}\left(\boldsymbol{l}_{\boldsymbol{\chi}}^{* *}\left(\boldsymbol{p}_{\boldsymbol{X}}\right)\right)=\prod_{l=1}^{L_{c}} \coprod_{k=1}^{r} \prod_{s \in B_{l, k}} \coprod_{i \in A_{k}} p_{i}^{z_{i}^{l, k, s}+1} .
$$

\section{Lower bounds for availabilities based on minimal path vectors and for unavailabilities based on minimal cut vectors, and corresponding upper bounds}

The following theorem shows that the lower bounds (5) and (8) are unchanged under a modular decomposition.

Theorem 2. Let $(\psi, \chi)$ be a modular decomposition of $(C, \phi)$ as in (3). Let the joint distributions of the independent performance processes $\boldsymbol{X}_{A_{k}}$ be arbitrary, $k=1, \ldots, r$. Let $j \in S$ be arbitrary. Comparing lower bounds for the system availability $p_{\phi}^{j}$ in an interval I based on $M P V s$, we then have

$$
l_{\phi}^{\prime \prime}=l_{\psi}^{\prime}\left(\mathbf{l}_{\chi}^{\prime \prime}\right) \leq p_{\phi}^{j}
$$

Moreover, with an arbitrary joint distribution for the performance process $\boldsymbol{X}=\left(X_{1}, \ldots, X_{n}\right)$, we also have the identity

$$
l_{\phi}^{\prime}\left(\boldsymbol{p}_{\boldsymbol{X}}\right)=l_{\psi}^{\prime}\left(\mathbf{l}_{\chi}^{\prime}\left(\boldsymbol{p}_{\boldsymbol{X}}\right)\right)
$$

representing a lower bound if the component processes are associated in I. Analogous results are valid for the corresponding lower unavailability bounds.

Proof. Let $\boldsymbol{y}$ be an MPV for $\phi$ to level $j$ for which the maximum in the definition of $l_{\phi}^{\prime \prime}$ is obtained. By Lemma 1(a)(i), choose an MPV $\boldsymbol{u}^{l}$ for $\psi$ to level $j$ such that $\chi_{k}\left(\boldsymbol{y}_{A_{k}}\right) \geq$ $u_{k}^{l}, k=1, \ldots, r$. Again, by Lemma 1(a)(i), using the notation of Definition 1, $\boldsymbol{y}$ is of the form $\boldsymbol{y}=\left(\boldsymbol{y}_{A_{1}}^{l, s_{1}}, \ldots, \boldsymbol{y}_{A_{r}}^{l, s_{r}}\right)$ for some $\boldsymbol{s}=\left(s_{1}, \ldots, s_{r}\right) \in E_{l, 1} \times \cdots \times E_{l, r}$. Owing to the independence of the modules we have

$$
\begin{aligned}
l_{\phi}^{\prime \prime} & =\prod_{k=1}^{r} \mathrm{P}\left(X_{i}(t) \geq y_{i}^{l, k, s_{k}} \text { for all } i \in A_{k}, t \in I\right) \\
& \leq \max _{1 \leq l \leq L_{p}} \prod_{k=1}^{r}\left[\max _{s_{k} \in E_{l, k}} \mathrm{P}\left(X_{i}(t) \geq y_{i}^{l, k, s_{k}} \text { for all } i \in A_{k}, t \in I\right)\right] \\
& =l_{\psi}^{\prime}\left(l_{\chi}^{\prime \prime}\right) .
\end{aligned}
$$

To prove the opposite inequality, choose $\boldsymbol{u}^{l}$ and $\left(\boldsymbol{y}_{A_{1}}^{l, s_{1}}, \ldots, \boldsymbol{y}_{A_{r}}^{l, s_{r}}\right)$ for which the right-hand side of (15) is attained. Then $\boldsymbol{y}=\left(\boldsymbol{y}_{A_{1}}^{l, s_{1}}, \ldots, \boldsymbol{y}_{A_{r}}^{l, s_{r}}\right)$ is a path vector for $\phi$ to level $j$. In case it is not minimal, choose an MPV $\boldsymbol{y}^{\prime} \leq \boldsymbol{y}$. Then

$$
\begin{aligned}
l_{\phi}^{\prime \prime} & \geq \mathrm{P}\left(X_{i}(t) \geq y_{i}^{\prime} \text { for all } i=1, \ldots, n, t \in I\right) \\
& =\prod_{k=1}^{r} \mathrm{P}\left(X_{i}(t) \geq y_{i}^{\prime} \text { for all } i \in A_{k}, t \in I\right) \\
& \geq \prod_{k=1}^{r} \mathrm{P}\left(X_{i}(t) \geq y_{i}^{l, k, s_{k}} \text { for all } i \in A_{k}, t \in I\right) \\
& =l_{\psi}^{\prime}\left(l_{\chi}^{\prime \prime}\right),
\end{aligned}
$$

where the last inequality follows since $y_{i}^{l, k, s_{k}} \geq y_{i}^{\prime}$ for all $i \in A_{k}, k=1, \ldots, r$. Hence, (13) is proved. 
If the component processes $X_{i}, i=1, \ldots, n$, are independent in $I$ then, as noted after (8), $l_{\phi}^{\prime \prime j}$ and $l_{\phi}^{\prime j}\left(\boldsymbol{p}_{\boldsymbol{x}}\right)$ coincide. Also, $l_{\chi_{k}}^{\prime \prime s}$ and $l_{\chi_{k}}^{\prime s}\left(\boldsymbol{p}_{\boldsymbol{x}}\right)$ coincide for each $k=1, \ldots, r$ and each $s \in S_{\chi_{k}}$. Hence, $\boldsymbol{l}_{\chi}^{\prime \prime}=\boldsymbol{l}_{\chi}^{\prime}\left(\boldsymbol{p}_{\boldsymbol{x}}\right)$ also. It follows that (17) holds in the case of independent component processes. Now let the performance process $\boldsymbol{X}=\left(X_{1}, \ldots, X_{n}\right)$ have an arbitrary joint distribution. According to (8), applied to $\phi, \psi$, and each $\chi_{k}, k=1, \ldots, r$, we can express the left- and right-hand sides of (14) explicitly in terms of the component availability vector $p_{x}=\left\{p_{i}^{s}, s \in S_{i}, i=1, \ldots, n\right\}$ as

$$
\begin{gathered}
l_{\phi}^{\prime}\left(\boldsymbol{p}_{\boldsymbol{x}}\right)=\max _{1 \leq m \leq M_{p}} \prod_{i=1}^{n} p_{i}^{y_{i}^{m}}=: f\left(\boldsymbol{p}_{\boldsymbol{x}}\right), \\
l_{\psi}^{\prime}\left(\boldsymbol{l}_{\boldsymbol{\chi}}^{\prime}\left(\boldsymbol{p}_{\boldsymbol{x}}\right)\right)=\max _{1 \leq l \leq L_{p}} \prod_{k=1}^{r}\left[\max _{s \in E_{l, k}} \prod_{i \in A_{k}} p_{i}^{y_{i}^{l, k, s}}\right]=: g\left(\boldsymbol{p}_{\boldsymbol{x}}\right) .
\end{gathered}
$$

We claim that the two functions $f$ and $g$ defined by (16) agree for any vector $\boldsymbol{p}_{\boldsymbol{x}}^{\prime}=\left\{p_{i}^{\prime s}, s \in S_{i}\right.$, $i=1, \ldots, n\}$ that satisfies the requirement that $p_{i}^{\prime s}$ is nonincreasing in $s$ for each $i=1, \ldots, n$. To see this, choose an arbitrary time point $t$, and define independent component state variables $X_{1}^{\prime}(t), \ldots, X_{n}^{\prime}(t)$, whose availabilities at $t$ are given by $\boldsymbol{p}_{x}^{\prime}$, i.e. $\mathrm{P}\left(X_{i}^{\prime}(t) \geq s\right)=p_{i}^{\prime s}$ for each $s$ and $i$. Since the variables $X_{i}^{\prime}(t)$ are independent, we can apply the theorem with $I$ replaced by $[t, t]$, and with the process $\boldsymbol{X}$ replaced by $\boldsymbol{X}^{\prime}(t)$. The conclusion is that $f\left(\boldsymbol{p}_{x}^{\prime}\right)=g\left(\boldsymbol{p}_{x}^{\prime}\right)$, proving our claim. However, it is obvious that the availabilities $p_{i}^{s}$ of the arbitrarily chosen process $\boldsymbol{X}$ also satisfy the requirement that $p_{i}^{s}$ is nonincreasing in $s$. It follows that $f\left(\boldsymbol{p}_{\boldsymbol{x}}\right)=g\left(\boldsymbol{p}_{\boldsymbol{x}}\right)$, and (14) is proved. The fact that (14) represents a lower bound if the component processes are associated in $I$ is, as already mentioned, proved in Funnemark and Natvig (1985). This completes the proof.

Theorem 2 shows that nothing is either gained or lost by using a modular decomposition in connection with the $l^{\prime}$ and $l^{\prime \prime}$ bounds in terms of the closeness to $p_{\phi}^{j}$. However, the modular decomposition may be advantageous from the computational point of view. Theorem 2 is also stated in Funnemark and Natvig (1985), but the proof uses the inadequate characterization of the MPVs of $\phi$ given in Butler (1982). The same holds for the comparison of upper availability bounds, originally presented in Funnemark and Natvig (1985) and Natvig (1986), given in Theorem 3 below. Although our main focus is on lower bounds, we include a corrected proof of this result here for the sake of completeness. Note that the upper bound $u_{\phi}^{\prime \prime}(I)$ appearing in the theorem is based on taking the minimum over the factors appearing in the lower bound (4) rather than their product.

Theorem 3. Let $(\psi, \chi)$ be a modular decomposition of $(C, \phi)$ as in (3). Let $j \in S$ be arbitrary, and consider the following upper bound for $p_{\phi}^{j}$ :

$$
u_{\phi}^{\prime \prime}(I)=\min _{1 \leq m \leq M_{c}} \mathrm{P}\left(\left[\max _{1 \leq i \leq n}\left(X_{i}(t)-z_{i}^{m}\right)\right]>0 \text { for all } t \in I\right) .
$$

We then have

$$
u_{\phi}^{\prime \prime}(I) \leq \inf _{t \in I}\left(1-\bar{l}_{\phi}^{\prime \prime}([t, t])\right)=\inf _{t \in I}\left(1-\bar{l}_{\psi}^{\prime}\left(\bar{l}_{\chi}^{\prime \prime}([t, t])\right)\right) .
$$

The corresponding result is valid for the upper bounds for unavailabilities to level $j$ based on MPVs. 
Proof. Choose an arbitrary $t \in I$. For any $\mathrm{MCV} z$ of $\phi$ to level $j$, we have

$$
\mathrm{P}\left(\left[\max _{1 \leq i \leq n}\left(X_{i}(t)-z_{i}\right)\right]>0\right)=\mathrm{P}\left(\bigcup_{i=1}^{n}\left(X_{i}(t)>z_{i}\right)\right)=1-\mathrm{P}\left(\bigcap_{i=1}^{n}\left(X_{i}(t) \leq z_{i}\right)\right) .
$$

Minimizing this over MCVs $z$ on both sides and using (9) yields

$$
u_{\phi}^{\prime \prime}([t, t])=1-\bar{l}_{\phi}^{\prime \prime}([t, t]) .
$$

We obviously have $u_{\phi}^{\prime \prime}(I) \leq u_{\phi}^{\prime \prime}([t, t])$. Hence, the stated inequality follows by taking the infimum over all $t \in I$. The subsequent equality follows by using the unavailability part of Theorem 2.

\section{Lower availability bounds based on minimal cut vectors}

In order to deal with the $l^{*}$ and $l^{* *}$ bounds of (4) and (7), we need the following lemma.

Lemma 2. Let $P^{k, s}, k=1, \ldots, r, s \in B_{k}=\left\{1, \ldots, b_{k}\right\}$ be real numbers between 0 and 1 . Then

$$
\prod_{s \in B_{1} \times \cdots \times B_{r}} \coprod_{k=1}^{r} P^{k, s_{k}} \leq \coprod_{k=1}^{r} \prod_{s \in B_{k}} P^{k, s} .
$$

Proof. If $b_{k}=1$ for each $k=1, \ldots, r$, the lemma is obviously true, since then both sides equal $\bigsqcup_{k=1}^{r} P^{k, 1}$. Hence, the lemma is true when $N=\sum_{k=1}^{r} b_{k}-r=0$. We prove the lemma by induction on $N$. We need the following inequality, valid for $p, q, w \in[0,1]$ :

$$
(p q) \coprod w-(p \coprod w)(q \coprod w) \geq 0
$$

Here, by definition, $p \bigsqcup q=1-(1-p)(1-q)=p+q-p q$. The inequality follows since

$$
\begin{aligned}
p q \amalg & w-(p \bigsqcup w)(q \coprod w) \\
& =(p q+w-p q w)-q(p+w-p w)-w(1-q)(p \coprod w) \\
& =w(1-q)-w(1-q)(p \coprod w) \\
& \geq 0 .
\end{aligned}
$$

With obvious interpretations of $p, q$, and $w$, this covers the special case $r=2, b_{1}=2$, and $b_{2}=1$ of the lemma. With the interpretation

$$
w=p^{2,1} \coprod \cdots \coprod p^{r, 1}
$$

(17) also covers the case $b_{1}=2, b_{2}=\cdots=b_{r}=1$, and arbitrary $r$. By symmetry, the lemma is true for $N=1$. Now assume that the lemma holds for some $N \geq 1$, and consider the case $N+1$. We may assume that $b_{1} \geq 2$. Define

$$
p=\prod_{s=1}^{b_{1}-1} P^{1, s}, \quad q=P^{1, b_{1}}, \quad \text { and } \quad w=\coprod_{k=2}^{r} \prod_{s \in B_{k}} P^{k, s} .
$$


Using (17) and the induction hypothesis, we then have

$$
\begin{aligned}
\coprod_{k=1}^{r} \prod_{s \in B_{k}} P^{k, s} & =p q \coprod w \\
& \geq(p \coprod w)(q \coprod w) \\
& \geq\left(\prod_{s \in\left(B_{1}-\left\{b_{1}\right\}\right) \times B_{2} \times \cdots \times B_{r}} \coprod_{k=1}^{r} P^{k, s_{k}}\right)\left(\prod_{s \in B_{2} \times \cdots \times B_{r}} P^{1, b_{1}} \coprod\left(\coprod_{k=2}^{r} P^{k, s_{k}}\right)\right) \\
& =\prod_{s \in B_{1} \times \cdots \times B_{r}} \coprod_{k=1}^{r} P^{k, s_{k}},
\end{aligned}
$$

proving the lemma.

In our analysis of the behaviour of the $l^{*}$ and $l^{* *}$ bounds, we have to start with the special case of an interval of the form $[t, t]$ for an arbitrary $t \in I$. For this special case, we introduce the following notation, which simplifies the mathematical expressions. Recalling from Definition 1 that the $z_{i}^{l, k, s}$ are the components of the MCVs $z_{A_{k}}^{l, s}, s \in B_{l, k}$, of $\chi_{k}$ to level $v_{k}^{l+}$, define

$$
P^{l, k, s}=\mathrm{P}\left(\bigcup_{i \in A_{k}}\left(X_{i}(t)>z_{i}^{l, k, s}\right)\right), \quad l=1, \ldots, L_{c}, k=1, \ldots, r, s \in B_{l, k} .
$$

Using this notation and replacing $\phi$ by $\chi_{k}$ in (4), we then have

$$
l_{\chi k}^{*\left(v_{k}^{l+}\right)}=\prod_{s \in B_{l, k}} P^{l, k, s}
$$

which is a component in the vector $\mathbf{l}_{\chi}^{*}$.

Theorem 4. Let $t \in I$. If the modular decomposition $(\psi, \chi)$ of $(C, \phi)$ has the property described in Theorem 1(iv), and, in particular, if the modular structure functions $\chi_{k}, k=$ $1, \ldots, r$, satisfy Theorem 1(iii) then

$$
l_{\phi}^{*}([t, t]) \leq l_{\psi}^{* *}\left(\mathbf{l}_{\chi}^{*}\right)([t, t]) .
$$

Proof. Using the property described in Theorem 1(iv), and then the independence of the modules and the simplifying notation (18), we have

$$
\begin{aligned}
l_{\phi}^{*}([t, t]) & =\prod_{l=1}^{L_{c}} \prod_{s \in B_{l, 1} \times \cdots \times B_{l, r}} \mathrm{P}\left(\bigcup_{k=1}^{r} \bigcup_{i \in A_{k}}\left(X_{i}(t)>z_{i}^{l, k, s_{k}}\right)\right) \\
& =\prod_{l=1}^{L_{c}} \prod_{s \in B_{l, 1} \times \cdots \times B_{l, r}} \coprod_{k=1}^{r} P^{l, k, s_{k}} .
\end{aligned}
$$

We now use Lemma 2 to each

$$
\prod_{s \in B_{l, 1} \times \cdots \times B_{l, r}} \coprod_{k=1}^{r} P^{l, k, s_{k}}, \quad l=1, \ldots, L_{c}
$$


of (20), and then use (19) to obtain

$$
l_{\phi}^{*}([t, t]) \leq \prod_{l=1}^{L_{c}} \coprod_{k=1}^{r} \prod_{s \in B_{l, k}} P^{l, k, s}=\prod_{l=1}^{L_{c}} \coprod_{k=1}^{r} l_{\chi_{k}}^{*\left(v_{k}^{l+}\right)}=l_{\psi}^{* *}\left(\mathbf{l}_{\chi}^{*}([t, t])\right),
$$

where the last equality follows by replacing $\phi$ by $\psi$ in (7). This completes the proof.

By Funnemark and Natvig (1985), Theorem 4 provides valid lower bounds for $p_{\phi}^{j}$ if the component states of each module, and, hence, all component states, at time $t$ are sets of associated random variables.

Attempting to extend (20) to the case of a general interval $I$ in place of $[t, t]$ leads to the inequality (cf. (4))

$$
\begin{aligned}
& \mathrm{P}\left(\left[\max _{1 \leq k \leq r} \max _{i \in A_{k}}\left(X_{i}(t)-z_{i}^{l, k, s}\right)\right]>0 \text { for all } t \in I\right) \\
& \quad \geq \mathrm{P}\left(\bigcup_{k=1}^{r}\left(\left[\max _{i \in A_{k}}\left(X_{i}(t)-z_{i}^{l, k, s}\right)\right]>0 \text { for all } t \in I\right)\right) \\
& \quad=\coprod_{k=1}^{r} \mathrm{P}\left(\left[\max _{i \in A_{k}}\left(X_{i}(t)-z_{i}^{l, k, s}\right)\right]>0 \text { for all } t \in I\right) .
\end{aligned}
$$

Since this inequality has the wrong direction, we are not able to generalize Theorem 4 to the case of a general interval. Roughly speaking, the explanation for this is that the expression on the left-hand side of the above inequality allows different components to prevent the cut vector from sabotaging the system at different times, while the expression on the right-hand side only allows different components within a single module to do so. However, specializing to the bounds based on independent components, we are finally able to obtain an extension to an arbitrary interval $I$.

Theorem 5. Let I be an arbitrary interval, and assume that the component processes are independent in I. If the modular decomposition $(\psi, \chi)$ of $(C, \phi)$ has the property described in Theorem 1(iv), and, in particular, if the modular structure functions $\chi_{k}, k=1, \ldots, r$, satisfy Theorem 1(iii) then

$$
l_{\phi}^{* *}\left(\boldsymbol{p}_{\boldsymbol{X}}\right) \leq l_{\psi}^{* *}\left(\mathbf{I}_{\boldsymbol{\chi}}^{* *}\left(\boldsymbol{p}_{\boldsymbol{X}}\right)\right)
$$

Proof. Consider first the case of a degenerate interval $[t, t]$. Since we are considering availabilities at a single point of time, and since the component states at this point of time are independent, the $l^{*}$ and $l^{* *}$ bounds coincide. Hence, the result follows by Theorem 4 . Comparing (20) and (21), this means that

$$
\prod_{l=1}^{L_{c}} \prod_{s \in B_{l, 1} \times \cdots \times B_{l, r}} \coprod_{k=1}^{r} \coprod_{i \in A_{k}} p_{i}^{l, k, s_{k}} \leq \prod_{l=1}^{L_{c}} \coprod_{k=1}^{r} \prod_{s \in B_{l, k}} \coprod_{i \in A_{k}} p_{i}^{l, k, s},
$$

where we have defined

$$
p_{i}^{l, k, s}=\mathrm{P}\left(X_{i}(t)>z_{i}^{l, k, s}\right) .
$$

But inequality (22) remains valid if $p_{i}^{l, k, s}$ is redefined as

$$
p_{i}^{l, k, s}=\mathrm{P}\left(X_{i}(t)>z_{i}^{l, k, s} \text { for all } t \in I\right)=p_{i}^{z_{i}^{l, k, s}+1} .
$$


Using the property described in Theorem 1(iv) and (7), the left-hand side of inequality (22) then becomes $l_{\phi}^{* * j}\left(\boldsymbol{p}_{\boldsymbol{X}}\right)$, while the right-hand side equals $l_{\psi}^{* * j}\left(\boldsymbol{l}_{\boldsymbol{\chi}}^{* *}\left(\boldsymbol{p}_{\boldsymbol{X}}\right)\right)$ by (12). This completes the proof.

The bounds of Theorems 2 and 5 can be combined to obtain a common improvement. Contrary to intuition, it is also the case that $l_{\phi}^{* * j}\left(\boldsymbol{p}_{\boldsymbol{x}}\right)$ is not necessarily nonincreasing in $j$ (consider for instance small values of $p$ in the cases $j=2$ and $j=3$ in Example 1). This motivates the maximization over $j^{\prime} \geq j$ in the definition of the lower bound

$$
B_{\phi}^{* j}\left(\boldsymbol{p}_{\boldsymbol{X}}\right)=\max _{j^{\prime} \geq j} \max \left(l_{\phi}^{* * j^{\prime}}\left(\boldsymbol{p}_{\boldsymbol{X}}\right), l_{\phi}^{j^{\prime}}\left(\boldsymbol{p}_{\boldsymbol{X}}\right)\right),
$$

introduced in Funnemark and Natvig (1985). As in Theorem 5, this bound may also be improved by modular decomposition. Applying Theorems 2 and 5 , as well as the facts that $B_{\psi}^{* j}(\cdot)$ is nondecreasing in each argument and that $p_{\phi}^{j}$ is nonincreasing in $j$, it is straightforward to prove the first inequality in the following corollary. For the second inequality, we also need the fact that $p_{\phi}^{j}=p_{\psi}^{j}$.

Corollary 1. Let I be an arbitrary interval, and assume that the component processes are independent in I. If the modular decomposition $(\psi, \chi)$ of $(C, \phi)$ has the property described in Theorem 1(iv), and, in particular, if the modular structure functions $\chi_{k}, k=1, \ldots, r$, satisfy Theorem 1(iii) then

$$
B_{\phi}^{*}\left(\boldsymbol{p}_{\boldsymbol{X}}\right) \leq B_{\psi}^{*}\left(\mathbf{B}_{\chi}^{*}\left(\boldsymbol{p}_{\boldsymbol{X}}\right)\right) \leq p_{\phi}^{j} .
$$

This result was given in Funnemark and Natvig (1985) for the case of $I=[t, t]$, but without realising the need for assumptions like those given in Theorem 1.

In this section, for simplicity, we have considered only lower availability bounds, although corresponding results for unavailability bounds, involving condition (i) and property (ii) of Theorem 1, can be given.

\section{Acknowledgements}

I am grateful to Professor Bent Natvig for inspiring me to look at the problems treated in this paper, and for his helpful comments and careful reading of the manuscript. I also want to thank an anonymous referee for having devoted time and energy to a thorough reading of this undeniably quite technical paper, as well as for his/her very useful suggestions.

\section{References}

Barlow, R. E. and Proschan, F. (1975). Statistical Theory of Reliability and Life Testing. Rinehart and Winston, New York.

Bodin, L. D. (1970). Approximations to system reliability using a modular decomposition. Technometrics 12, $335-344$.

Butler, D. A. (1982). Bounding the reliability of multistate systems. Operat. Res. 30, 530-544.

Esary, J. D. and Proschan, F. (1970). A reliability bound for systems of maintained, interdependent components. J. Amer. Statist. Assoc. 65, 329-338.

Funnemark, E. and Natvig, B. (1985). Bounds for the availabilities in a fixed time interval for multistate monotone systems. Adv. Appl. Prob. 17, 638-665.

Gåsemyr, J. and Natvig, B. (2005). Probabilistic modelling of monitoring and maintenance of multistate monotone systems with dependent components. Methodology Comput. Appl. Prob. 7, 63-78.

Huseby, A. et al. (2010). Advanced discrete event simulation methods with application to importance measure estimation in reliability. In Discrete Event Simulations, ed. A. Goti, InTech, 18pp.

Huseby, A. B. and Natvig, B. (2010). Advanced discrete simulation methods applied to repairable multistate systems. In Reliability, Risk and Safety. Theory and Applications, eds R. Bris, S. Martorell and C. Guedes Soares, CRC Press, pp. 659-666. 
Natvig, B. (1980). Improved bounds for the availability and unavailability in a fixed time interval for systems of maintained, interdependent components. Adv. Appl. Prob. 12, 200-221.

Natvig, B. (1986). Improved upper bounds for the availabilities in a fixed time interval for multistate monotone systems. Adv. Appl. Prob. 18, 577-579.

Natvig, B. (1993). Strict and exact bounds for the availabilities in a fixed time interval for multistate monotone systems. Scand. J. Statist. 20, 171-175.

Natvig, B. (2011). Multistate Systems Reliability Theory with Applications. John Wiley, Chichester.

Natvig, B. and Mørch, H. W. H. (2003). An application of multistate reliability theory to an offshore gas pipeline network. Internat. J. Reliab. Quality Safety Eng. 10, 361-381. 